\title{
LIMITS OF LARGE METAPOPULATIONS WITH PATCH-DEPENDENT EXTINCTION PROBABILITIES
}

\author{
R. MCVINISH ${ }^{* * *}$ AND \\ P. K. POLLETT, ${ }^{* * *}$ University of Queensland
}

\begin{abstract}
We propose a model for the presence/absence of a population in a collection of habitat patches. This model assumes that colonisation and extinction of the patches occur as distinct phases. Importantly, the local extinction probabilities are allowed to vary between patches. This permits an investigation of the effect of habitat degradation on the persistence of the population. The limiting behaviour of the model is examined as the number of habitat patches increases to $\infty$. This is done in the case where the number of patches and the initial number of occupied patches increase at the same rate, and for the case where the initial number of occupied patches remains fixed.
\end{abstract}

Keywords: Chain binomial model; fixed point; weak convergence; point process

2010 Mathematics Subject Classification: Primary 60J20

Secondary 60G55; 60F05

\section{Introduction}

Many species exist as a collection of local populations occupying spatially distinct patches. Ecologists refer to such collections of populations as metapopulations (see [13] for a review). Metapopulations are constantly changing due to the processes of extinction and colonisation occurring at each patch. For ecologists, the primary concern is the persistence of the metapopulation since, in the absence of an external source of migrants, there is a significant risk that the metapopulation will become extinct. This concern for the survival of the metapopulation as a whole is heightened by the presence of habitat destruction and degradation.

Metapopulation models provide the ecologist with tools for better understanding metapopulation dynamics and the effects of habit changes. While a number of metapopulation models have been proposed, perhaps the most widely applied models belong to the class of stochastic patch occupancy models (SPOMs). Hanski and Ovaskainen [14] provided a review of the SPOM literature up to 2003. The characterising feature of a SPOM is that only the presence/absence of a population at each patch is modelled. The size and age structure of each patch's population are ignored. Employing the Markov assumption, a SPOM can be mathematically described as a Markov chain whose state space comprises $2^{n}$ states for a metapopulation of $n$ patches.

SPOMs vary greatly in detail and realism. At one extreme, a simple SPOM might assume that all patches are identical and permit the movement of individuals from one patch to any other. Metapopulations of this type can often be modelled using the stochastic logistic model [22]. Owing to its simplicity, researchers have been able to derive a number of important properties of the model, such as the quasistationary distribution [17] and time until extinction [2]. At the

Received 2 December 2009; revision received 20 July 2010.

* Postal address: Department of Mathematics, University of Queensland, Brisbane, QLD 4072, Australia.

** Email address: r.mcvinish@uq.edu.au

*** Email address: pkp@maths.uq.edu.au 
other extreme, a SPOM might explicitly include a number of features of the environment, such as connectivity of patches, distances between patches, and the size and quality of patches (see [21] for an application to a tree frog metapopulation). The complexity of these models typically means that their properties need to be studied using simulation [16]. However, some analytical progress has been made for a spatial Levins metapopulation model on a random landscape in [8] and [18].

The model studied in this paper lies between these two extremes. We assume a simplified colonisation process where the probability of an unoccupied patch being colonised depends only on the number of occupied patches in the metapopulation. The connectivity and distance between patches are ignored. However, we permit heterogeneity among patches by allowing the probability of local population extinction to be patch dependent. In this way, we are able to incorporate habitat quality in the model which will assist our understanding of the effect of habitat degradation on metapopulation survival.

For each $n$, let $\left\{X_{t}^{n}\right\}_{t=1}^{T}$ be a discrete time-homogeneous Markov chain, where

$$
X_{t}^{n}=\left(X_{1, t}^{n}, \ldots, X_{n, t}^{n}\right) \quad \text { with } \quad X_{i, t}^{n}= \begin{cases}1 & \text { if patch } i \text { is occupied at time } t \\ 0 & \text { otherwise }\end{cases}
$$

We assume that colonisation and extinction occur in two distinct phases and that these two phases alternate as in the models studied in [5] and [15]. During the colonisation phase, a patch that is unoccupied at time $t$ will become occupied with probability $f\left(n^{-1} \sum_{j} X_{j, t}^{n}\right)$, where $f:[0,1] \mapsto[0,1]$. Letting $\tilde{X}_{t}^{n}=\left(\tilde{X}_{1, t}^{n}, \ldots, \tilde{X}_{n, t}^{n}\right)$ denote the state of the metapopulation after colonisation at time $t$, we may express the colonisation phase as

$$
\tilde{X}_{i, t}^{n} \sim X_{i, t}^{n}+B\left(1-X_{i, t}^{n}, f\left(n^{-1} \sum_{j=1}^{n} X_{j, t}^{n}\right)\right),
$$

where $B$ denotes a binomial distribution. It seems biologically reasonable to expect that an increase in the number of occupied patches will lead to an increase in the probability of an unoccupied patch becoming occupied during colonisation. Furthermore, typical colonisation functions, such as those used in [15], have the property that the colonisation probability increases by less with each additional occupied patch. In other words, the colonisation function is typically concave. Finally, it is assumed that $f^{\prime}(0)>0$. This excludes the case where $f(x)=0$ for all $x \in[0,1]$. We group these conditions on $f$ as hypothesis (A):

(A) The colonisation function $f:[0,1] \mapsto[0,1]$ is an increasing, concave function such that $f^{\prime}(0)>0$.

During the extinction phase, an occupied patch will become unoccupied with probability $e_{i}>0$, independently of the other patches. The probability of patch extinction may be affected by a number of variables, including patch size, prevalence of disease, and availability of food. Together, these variables describe the quality of the patch which will be measured by the probability of extinction. Denoting the patch survival probability by $s_{i}=1-e_{i}$, we may express the extinction phase as

$$
X_{i, t+1}^{n} \sim B\left(\tilde{X}_{i, t}^{n}, s_{i}\right)
$$

Although we assume that the metapopulation is observed only after the extinction phase, there is no compelling reason for this assumption. The results could have also been derived for 
observations taken after the colonisation phase. Finally, we note that the above model has much in common with the chain-binomial models used to model epidemics [3], [10], [12].

The aim of this paper is to study this process when the number of patches in the metapopulation increases to $\infty$. In Section 2 we show that the proportion of occupied patches in the metapopulation converges to the solution of a system of difference equations. The fixed points of the difference equation are identified and conditions under which these fixed points are stable are given. In Section 3 we study the behaviour of the metapopulation when only a small number of patches are occupied initially. We show that as the number of habitat patches increases to $\infty$, the set of occupied patches can be viewed as a point process on $[0,1)$ with the locations being given by the corresponding patch survival probabilities. We then proceed to calculate the probability of the metapopulation going extinct. We conclude with a brief discussion of the impact of habitat degradation on the survival of the metapopulation and the possible extension of the model and analysis to the continuous-time setting.

\section{Deterministic limit}

Given the complexity of the model, we shall study the behaviour of the metapopulation through asymptotic analysis. In this section we focus on the proportion of occupied patches in the metapopulation and establish convergence to a deterministic quantity. This approach to studying Markov chains has a broad literature. Darling and Norris [11] provided a recent survey of the literature and also determined some simple conditions under which a continuous-time Markov chain may be approximated by the solution to a differential equation. For our model, we show that on finite-time intervals the proportion of occupied patches converges as $n \rightarrow \infty$ to the solution to a system of difference equations. Let $\sigma$ denote a probability measure on $[0,1)$, and let $\bar{s}_{k}$ denote the $k$ th moment of $\sigma$, that is,

$$
\bar{s}_{k}=\int_{0}^{1} \lambda^{k} \sigma(\mathrm{d} \lambda)
$$

Since $\sigma$ is supported on $[0,1), \bar{s}_{k}$ exists for all $k=0,1,2, \ldots$

Theorem 2.1. Suppose that there exist a probability measure $\sigma$ and deterministic sequence $\{d(0, k)\}$ such that

$$
n^{-1} \sum_{i=1}^{n} s_{i}^{k} \stackrel{\mathrm{P}}{\rightarrow} \bar{s}_{k} \quad \text { and } \quad n^{-1} \sum_{i=1}^{n} s_{i}^{k} X_{i, 0}^{n} \stackrel{\mathrm{P}}{\rightarrow} d(0, k)
$$

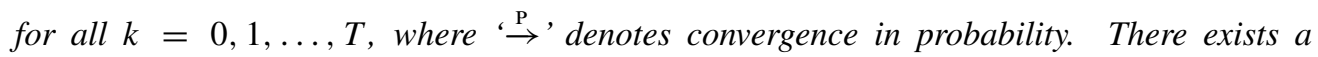
deterministic triangular arrayd $(t, k)$ such that, for all $t=0,1, \ldots, T$ and $k=0,1, \ldots, T-t$,

$$
n^{-1} \sum_{i=1}^{n} s_{i}^{k} X_{i, t}^{n} \stackrel{\mathrm{P}}{\rightarrow} d(t, k)
$$

where

$$
d(t+1, k)=d(t, k+1)+f(d(t, 0))\left(\bar{s}_{k+1}-d(t, k+1)\right) .
$$

Typically, we are only interested in $d(t, 0)$, which is the asymptotic proportion of occupied patches. However, we may still interpret the ratio $d(t, k) / d(t, 0), k \geq 1$, as the $k$ th moment of the conditional distribution of the patch survival probability given that the corresponding patch is occupied. From these moments, the conditional distribution could then be reconstructed. 
When $\bar{s}_{k}=\bar{s}_{1}^{k}$ for all $k$, that is, the patch survival probabilities are all equal, then it is possible to simplify (2.1). We can show by induction that $d(t, k)=\bar{s}_{1}^{k} x_{t}$ and

$$
x_{t+1}=\bar{s}_{1}\left(x_{t}+f\left(x_{t}\right)\left(1-x_{t}\right)\right) .
$$

If $f(x)=c x$ for some $c \in(0,1)$ then the resulting recursion can be viewed as a discrete-time approximation to the classical Levins model [13, p. 74]. It can be shown that if $c \leq\left(1-\bar{s}_{1}\right) / \bar{s}_{1}$ then 0 is the stable fixed point of (2.2), and if $c>\left(1-\bar{s}_{1}\right) / \bar{s}_{1}$ then

$$
x_{e q}=1-\frac{1-\bar{s}_{1}}{c \bar{s}_{1}}
$$

is the stable fixed point of (2.2) [6]. Fixed points and their stability are important because they indicate if the metapopulation will tend to persist and at what level. We now consider the fixed points of (2.1) and their stability.

Theorem 2.2. Assume that the colonisation function satisfies hypothesis (A). The fixed points of the recursion (2.1) are given by

$$
d(k)=\int_{0}^{1} \frac{f(\psi) \lambda^{k+1}}{1-\lambda+f(\psi) \lambda} \sigma(\mathrm{d} \lambda),
$$

where $\psi$ solves

$$
R(\psi)=\int_{0}^{1} \frac{f(\psi) \lambda}{1-\lambda+f(\psi) \lambda} \sigma(\mathrm{d} \lambda)=\psi
$$

If $f(0)>0$ then there exists a unique $\psi>0$ satisfying (2.3). If $f(0)=0$ and

$$
f^{\prime}(0) \int_{0}^{1} \frac{\lambda}{1-\lambda} \sigma(\mathrm{d} \lambda) \leq 1,
$$

then $\psi=0$ is the unique solution to (2.3). Otherwise, (2.3) has two solutions, of which one is $\psi=0$.

Theorem 2.3. Assume that the colonisation function satisfies hypothesis (A). If $f(0)=0$ and inequality (2.4) is satisfied, then $d(k) \equiv 0$ is a stable fixed point of (2.1). Otherwise, the nonzero solution of (2.3) is a stable fixed point of (2.1).

The first point to note about the above result is that if $f$ is concave then $\psi$ and, hence, $d(k)$ are continuous functionals of $\sigma$. In other words, a small change to the quality of the habitat will only have a small effect on the persistence level of the metapopulation. Now consider two metapopulations whose respective patch survival probability distributions are $\sigma$ and $\sigma^{\prime}$. Assume that $\sigma^{\prime} \geq_{\text {st }} \sigma$, where ' $\geq_{\text {st }}$ ' denotes the usual stochastic ordering. As the integrand in (2.3) is increasing in $\lambda$, it follows that if $\sigma^{\prime} \geq_{\text {st }} \sigma$ then $R_{\sigma^{\prime}}(\psi) \geq R_{\sigma}(\psi)$. Hence, the corresponding nonzero fixed points satisfy $\psi_{\sigma^{\prime}} \geq \psi_{\sigma}$. This leads to the unsurprising conclusion that a decrease in the survival probabilities leads to a decrease in the average number of patches occupied in the metapopulation. However, when comparing two metapopulations, the stochastic ordering of the survival probabilities is important. Suppose that $\sigma$ and $\sigma^{\prime}$ are the patch survival probability distributions of two metapopulations with respective means $\bar{s}_{1}$ and $\bar{s}_{1}^{\prime}$. If the two distributions do not satisfy the stochastic ordering then it is possible for the respective solutions to (2.3) to satisfy $\psi_{\sigma^{\prime}}<\psi_{\sigma}$, although $\bar{s}_{1}<\bar{s}_{1}^{\prime}$. 


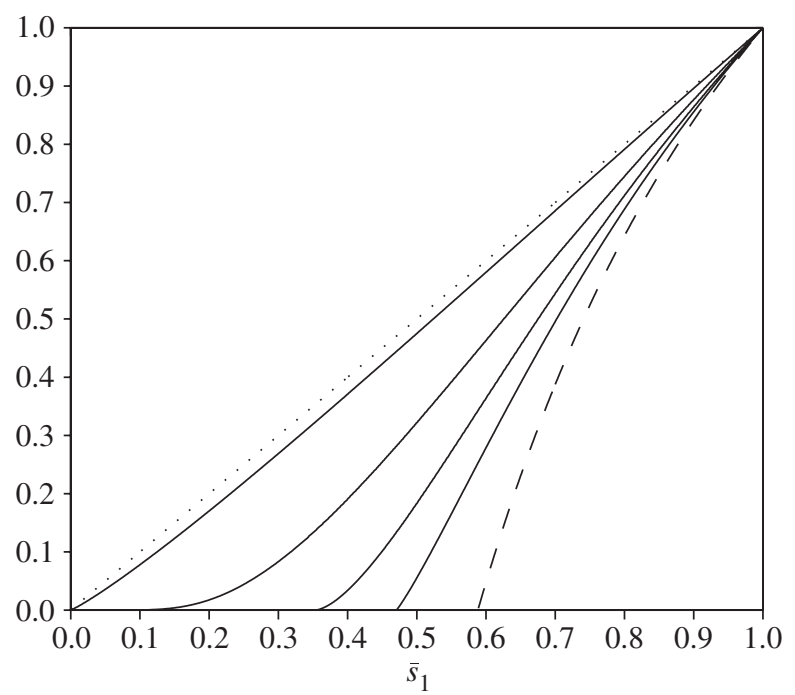

Figure 1: The solution to (2.5), where $f(x)=0.7 x$ is plotted against $\bar{s}_{1}$ for several values of $\gamma$. The dotted line corresponds to the limit as $\gamma \rightarrow 0$. From left to right, the four solid lines correspond to $\gamma=0.1,1,2.5$, and 5. The dashed line is the limiting case of $\gamma \rightarrow \infty$.

As an example, suppose that $\sigma$ is the beta distribution with parameters $\left(\bar{s}_{1} \gamma,\left(1-\bar{s}_{1}\right) \gamma\right)$. Assume that the colonisation function is not identically zero. Inequality (2.4) can be expressed as $f^{\prime}(0) \bar{s}_{1} \gamma>\left(1-\bar{s}_{1}\right) \gamma-1$ and the equilibrium point is given by the solution to

$$
\psi=\bar{s}_{1} f(\psi)_{2} F_{1}\left(1,1+\bar{s}_{1} \gamma ; 1+\gamma ; 1-f(\psi)\right),
$$

where ${ }_{2} F_{1}$ is Gauss' hypergeometric function [1, Chapter 15]. From a special case of the hypergeometric function [1, Equation (15.1.8)], we see that, as $\gamma \rightarrow 0, \psi \rightarrow \bar{s}_{1}$ for any colonisation function. In Figure 1 we plot the equilibrium points of a limiting metapopulation with colonisation function $f(x)=0.7 x$ against $\bar{s}_{1}$ for several values of $\gamma$. From the figure, it appears that the equilibrium point is decreasing as a function of $\gamma$ for a given $\bar{s}_{1}$.

Remark 2.1. The dynamics of the metapopulation when $f$ is not concave can be very sensitive to the patch survival probabilities. Suppose that $\sigma$ has distribution function $H\left(\lambda-\lambda_{0}\right)$, where $H$ is the unit step function. Define the colonisation function $f(x)$ as

$$
f(x)=\left(\frac{x\left(1-\lambda_{0}\right)}{(1-x) \lambda_{0}} \wedge 1\right) .
$$

This colonisation function is continuous, monotone increasing, and satisfies $f(0)=0$. Direct substitution shows that every $\psi \in\left[0, \lambda_{0}\right]$ is an equilibrium point of (2.2). Changes to the metapopulation which may only have a small effect on patch survival probabilities can have a catastrophic effect on the survival of the metapopulation as a whole. Let $\sigma^{\prime}$ have distribution function $H\left(\lambda-\lambda_{0}^{\prime}\right)$. If $\lambda_{0}^{\prime}>\lambda_{0}$ then the system (2.1) has a unique nonzero equilibrium $\psi=\lambda_{0}^{\prime}$. However, if $\lambda_{0}^{\prime}<\lambda_{0}$ then the unique equilibrium point of the system (2.1) is $\psi=0$. 


\section{Point process approximation}

We again examine the limiting behaviour of the Markov chain $X_{t}^{n}$; however, we shall now assume that $X_{0}^{n}$ is fixed for all $n$. In other words, we begin with a fixed number of occupied patches and we let the metapopulation increase as $n \rightarrow \infty$ by including additional unoccupied patches. By examining the limiting process we aim to determine conditions under which a metapopulation that is close to extinction may recover with positive probability. If every patch had the same patch survival probability, or one of a finite number of possibilities, then we might expect the number of occupied patches to converge to a (multitype) Galton-Watson process as $n \rightarrow \infty$. The limiting process could be studied using similar techniques to those used in [3]. We adopt a different approach in order to deal with the heterogeneity of the patch survival probabilities.

In order to address this problem, we treat the collection of patch survival probabilities of occupied patches at time $t$ as a point process on $[0,1)$. Let $S_{t}^{n}=\left\{s_{i}: X_{i, t}^{n}=1\right\}$. The probability generating functional of $S_{t}^{n}$ is defined by

$$
G_{S_{t}^{n}}(\xi)=\mathrm{E}\left(\prod_{s_{i} \in S_{t}^{n}} \xi\left(s_{i}\right)\right),
$$

where $\xi$ is a Borel function such that $0 \leq \xi(x) \leq 1$ for all $x \in[0,1)$ [9, Definition 9.4.IV]. The probability generating functional uniquely determines the point process [9, Theorem 9.4.V]. As $n \rightarrow \infty$, the sequence of point processes $S_{t}^{n}$ converges weakly to a point process $S_{t}$.

Theorem 3.1. As $n \rightarrow \infty$, $S_{t}^{n}$ converges weakly to $S_{t}$. The probability generating functional of $S_{t}$ is given by the recursion

$$
G_{S_{t+1}}(\xi)=G_{S_{t}}\left((1-x+x \xi) \exp \left(-f^{\prime}(0) \int_{0}^{1}(1-\xi(\lambda)) \lambda \sigma(\mathrm{d} \lambda)\right)\right) .
$$

The proof of Theorem 3.1 proceeds along the following argument. From (1.1) we might expect that if the number of occupied patches is fixed and the number of patches in the metapopulation is allowed to increase to $\infty$, then the number of newly colonised patches might converge to a Poisson random variable. As the colonisation probability does not depend on the patch survival probability, the collection of newly occupied patches after a colonisation phase might be well approximated by a nonhomogeneous Poisson process on $[0,1)$ with intensity measure proportional to $\sigma$. Also, from (1.2), the extinction phase closely resembles a thinning operation on the collection of occupied patches with thinning function $s$.

Extinction of the metapopulation by time $t$ corresponds to the event that $S_{t}$ is the empty set. This probability can be calculated from the probability generating functional in the same way that the probability of extinction of a branching process can be calculated from the probability generating function of the offspring. Let $\zeta_{b}(x)=b$ for all $x \in[0,1]$. Then

$$
\mathrm{P}\left(S_{t}=\varnothing\right)=\lim _{b \downarrow 0} G_{S_{t}}\left(\zeta_{b}\right) .
$$

Theorem 3.2. The sequence of point processes $\left\{S_{t}\right\}_{t=0}^{\infty}$ converges in distribution to the empty set with probability 1 if inequality (2.4) holds. Otherwise, the sequence of point processes $\left\{S_{t}\right\}$ converges to the empty set with probability

$$
G_{S_{0}}\left(\frac{\psi_{\infty}(1-x)}{1-\psi_{\infty} x}\right)
$$


where $\psi_{\infty}<1$ is the solution to

$$
\psi=\exp \left(-f^{\prime}(0) \int_{0}^{1} \frac{(1-\psi) \lambda}{1-\psi \lambda} \sigma(\mathrm{d} \lambda)\right) .
$$

When the patch survival probabilities are the same for each patch, we may use (3.2) to express $\psi_{\infty}$ in terms of the probability of $S_{t}$ converging to the empty set. Substituting this expression of $\psi_{\infty}$ into (3.3) gives the standard branching process result.

Consider again the case where $\sigma$ is a beta distribution with parameters $\left(\bar{s}_{1} \gamma,\left(1-\bar{s}_{1}\right) \gamma\right)$. With this choice of $\sigma,(3.3)$ becomes

$$
\psi=\exp \left(-\bar{s}_{1} f^{\prime}(0)(1-\psi){ }_{2} F_{1}\left(1,1+\bar{s}_{1} \gamma ; 1+\gamma ; \psi\right)\right) .
$$

Assume that $X_{0}$ comprises a single occupied habitat patch with survival probability $\bar{s}_{1}$. Equivalently, $S_{0}$ comprises a single point at $\bar{s}_{1}$. If $\gamma \rightarrow \infty$ then the probability of the limiting metapopulation going extinct is given by the branching process result since $\sigma$ concentrates at $\bar{s}_{1}$. On the other hand, if $\gamma \rightarrow 0$ then, from a special case of the hypergeometric function [1, Equation (15.1.8)], we see that the probability of the limiting metapopulation going extinct approaches

$$
\frac{\left(1-\bar{s}_{1}\right) \exp \left(-f^{\prime}(0) \bar{s}_{1}\right)}{1-\bar{s}_{1} \exp \left(-f^{\prime}(0) \bar{s}_{1}\right)} .
$$

Equation (3.4) was solved for $\psi$ taking $f^{\prime}(0)=0.7$ and the solution was then substituted into (3.2) to obtain the probability of extinction. In Figure 2 we plot the probability of the limiting metapopulation going extinct against $\bar{s}_{1}$ for several values of $\gamma$. It appears from this graph that the extinction probability is increasing in $\gamma$ for a fixed $\bar{s}_{1}$.

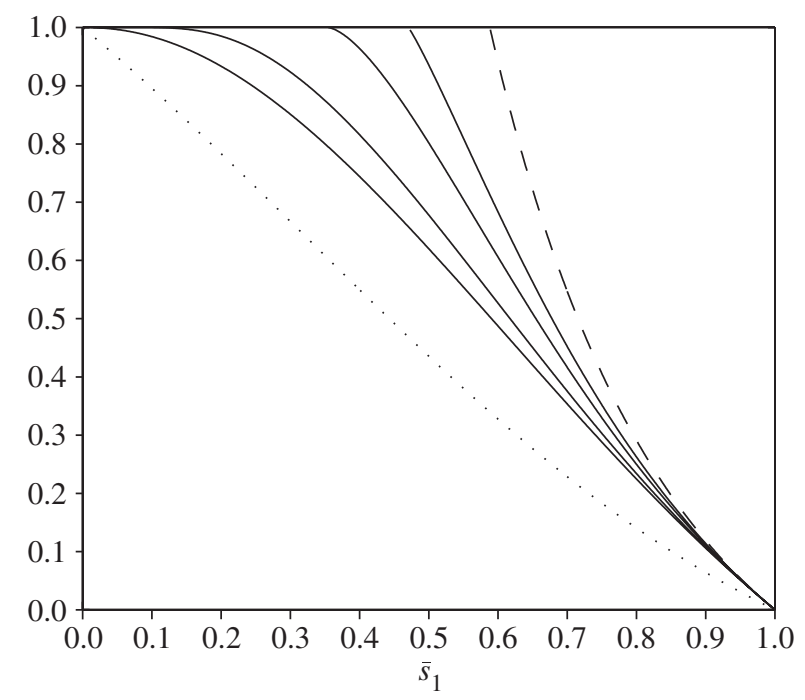

FIGURE 2: The probability of extinction of the limiting metapopulation is plotted against $\bar{s}_{1}$ for several values of $\gamma$. The dotted line corresponds to the limit as $\gamma \rightarrow 0$. From left to right, the four solid lines correspond to $\gamma=0.5,1,2.5$, and 5. The dashed line is the limiting case of $\gamma \rightarrow \infty$. 


\section{Discussion}

The results of this paper demonstrate that the persistence of a metapopulation crucially depends on the quality of the habitat patches. Inequality (2.4) provides the persistence criteria for a metapopulation with a large number of patches. It is also the condition that a large metapopulation must satisfy in order to have a positive probability of recovering from near extinction. The form of the integrand in (2.4) suggests that a patch with a survival probability of $\frac{2}{3}$ contributes twice as much to the survival of the metapopulation as a patch with a survival probability of $\frac{1}{2}$, and a patch with a survival probability of $\frac{9}{10}$ is approximately ten times as valuable as a patch with a survival probability of $\frac{1}{2}$. The importance of a high quality habitat was further highlighted in the numerical examples. Although this model has made many simplifications concerning the colonisation phase, we believe it is still a useful model for understanding the effects of habitat destruction on the metapopulations survival. Empirical studies show that, at least for some species, the connectivity of habitat patches has only a small effect on the colonisation patterns in the metapopulation [19].

Finally, it is natural to consider how this type of model could be handled in a continuous-time setting. Let $X_{t}^{n}$ now be a continuous-time homogeneous Markov chain. If $X_{i, t}^{n}=1$, that is, if patch $i$ is occupied at time $t$, then the population at patch $i$ goes extinct at a rate $\gamma_{i}>0$. Otherwise, if patch $X_{i, t}^{n}=0$, that is, if patch $i$ is unoccupied at time $t$, then it is colonised at a rate $c n^{-1} \sum_{i=1}^{n} X_{i, t}$ for some $c>0$. This is a special case of a more general continuous-time metapopulation model discussed in [7, Chapter 3]. Note that if $\gamma_{i}=\gamma$ for all $i$ then the model essentially reduces to the stochastic logistic model. If the initial number of occupied patches is allowed to increase at the same rate as the total number of patches, then we might expect that the average proportion of occupied patches to converge to a deterministic limit. To establish this limit, we could construct the associated process $Y^{n}(t)$, where

$$
Y_{j}^{n}(t)=n^{-1} \sum_{i=1}^{n} \gamma_{i}^{j} X_{i, t}^{n}
$$

for all $j=0,1,2, \ldots$ It seems plausible that weak convergence of $Y^{n}(t)$ to the solution of an infinite system of ordinary differential equations could then be established using arguments similar to those used in [4]. Alternatively, if the number of occupied patches remains fixed as $n \rightarrow \infty$, then the limiting behaviour of the set $\Gamma_{t}^{n}:=\left\{\gamma_{i}: X_{i, t}^{n}=1\right\}$ could be of interest. Intuitively, we would expect $\Gamma_{t}^{n}$ to converge weakly to a spatial birth-death process [20]. Both of these limits will be investigated in future work.

\section{Appendix A. Proofs of the theorems}

\section{A.1. Proof of Theorem 2.1}

The proof proceeds by induction on $t$. By assumption, $n^{-1} \sum_{i=1}^{n} s_{i}^{k} X_{i, 0}^{n} \stackrel{\mathrm{P}}{\rightarrow} d(0, k)$ for $k=0, \ldots, T$. Suppose that $n^{-1} \sum_{i=1}^{n} s_{i}^{k} X_{i, t}^{n} \stackrel{\mathrm{P}}{\rightarrow} d(t, k)$ for all $k=0, \ldots, T-t$. The state of the metapopulation after the colonisation phase is

$$
\tilde{X}_{i, t+1}^{n} \stackrel{\mathrm{D}}{=} X_{i, t}^{n}+\left(1-X_{i, t}^{n}\right) Z_{i},
$$

where, conditional on $X_{t}^{n}$, the $Z_{i}$ are independent Bernoulli random variables with probability of success $f n^{-1} \sum_{i=1}^{n} X_{i, t}^{n}$. Here ' $=$, denotes equality in distribution. We aim to show that $n^{-1} \sum_{i=1}^{n} s_{i}^{k} \tilde{X}_{i, t+1}^{n}$ converges in probability to some deterministic value for each 


$$
\begin{aligned}
& k=0,1, \ldots, T-t: \\
& \mathrm{E}\left(n^{-1} \sum_{i=1}^{n} s_{i}^{k} \tilde{X}_{i, t+1}^{n} \mid X_{t}^{n}\right) \\
& \quad=n^{-1} \sum_{i=1}^{n} s_{i}^{k} X_{i, t}^{n}+f\left(n^{-1} \sum_{j=1}^{n} X_{j, t}^{n}\right)\left(n^{-1} \sum_{i=1}^{n} s_{i}^{k}-n^{-1} \sum_{i=1}^{n} s_{i}^{k} X_{i, t}^{n}\right) \\
& \quad \stackrel{\mathrm{P}}{\rightarrow} d(t, k)+f(d(t, 0))\left(\bar{s}_{k}-d(t, k)\right) \\
& \quad=: \tilde{d}(t, k), \\
& \operatorname{var}\left(n^{-1} \sum_{i=1}^{n} s_{i}^{k} \tilde{X}_{i, t+1}^{n} \mid X_{t}^{n}\right) \\
& \quad=n^{-2} \sum_{i=1}^{n} s_{i}^{2 k}\left(1-X_{i, t}^{n}\right) f\left(n^{-1} \sum_{j=1}^{n} X_{j, t}^{n}\right)\left(1-f\left(n^{-1} \sum_{j=1}^{n} X_{j, t}^{n}\right)\right) \\
& \quad \leq(4 n)^{-1} .
\end{aligned}
$$

Since $\tilde{d}(t, k)$ is deterministic, convergence in probability of $n^{-1} \sum_{i=1}^{n} s_{i}^{k} \tilde{X}_{i, t+1}^{n}$ to $\tilde{d}(t, k)$ now follows from (A.1), (A.2), and an application of Chebyshev's inequality. The state of the metapopulation after the extinction phase is

$$
X_{i, t+1}^{n} \stackrel{\mathrm{D}}{=} \tilde{X}_{i, t+1}^{n} W_{i},
$$

where $W_{i}$ are independent Bernoulli random variables with probability of success $s_{i}$. We now aim to show that $n^{-1} \sum_{i=1}^{n} s_{i}^{k} X_{i, t+1}^{n}$ converges in probability to some deterministic value for each $k=0,1, \ldots, T-(t+1)$ :

$$
\begin{aligned}
\mathrm{E}\left(n^{-1} \sum_{i=1}^{n} s_{i}^{k} X_{i, t+1}^{n} \mid \tilde{X}_{t+1}^{n}\right) & =n^{-1} \sum_{i=1}^{n} s_{i}^{k+1} \tilde{X}_{i, t+1}^{n} \\
& \stackrel{\mathrm{P}}{\rightarrow} \tilde{d}(t, k+1) \\
& =d(t, k+1)+f(d(t, 0))\left(\bar{s}_{k+1}-d(t, k+1)\right),
\end{aligned}
$$

where (A.3) follows from the convergence in probability of $n^{-1} \sum_{i=1}^{n} s_{i}^{k+1} \tilde{X}_{i, t+1}^{n}$ and (A.1),

$$
\operatorname{var}\left(n^{-1} \sum_{i=1}^{n} s_{i}^{k} X_{i, t+1}^{n} \mid \tilde{X}_{t+1}^{n}\right)=n^{-2} \sum_{i=1}^{n} s_{i}^{2 k+1}\left(1-s_{i}\right) \tilde{X}_{i, t+1}^{n} \leq(4 n)^{-1} .
$$

Again, since $d(t, k)$ is deterministic, convergence in probability of $n^{-1} \sum_{i=1}^{n} s_{i}^{k} X_{i, t+1}^{n}$ to $d(t+$ $1, k$ ) follows from (A.3), (A.4), and an application of Chebyshev's inequality.

\section{A.2. Proof of Theorem 2.2}

The fixed points of (2.1) solve

$$
d(k)=d(k+1)+f(d(0))\left(\bar{s}_{k+1}-d(k+1)\right) .
$$

In order to solve (A.5), we solve the linear recursion

$$
\bar{d}(k)=(1-f(\psi)) \bar{d}(k+1)+f(\psi) \bar{s}_{k+1},
$$


subject to $\bar{d}(0)=\psi$. As the sequence $d(t, k)$ is completely monotone in $k, d(k)$ must also be completely monotone. We now write $\bar{d}(k)=\int_{0}^{1} \lambda^{k} \mu(\mathrm{d} \lambda)$ for some Borel measure $\mu$. Substituting this expression into (A.6) gives

$$
\int_{0}^{1} \lambda^{k} \mu(\mathrm{d} \lambda)=(1-f(\psi)) \int_{0}^{1} \lambda^{k+1} \mu(\mathrm{d} \lambda)+f(\psi) \int_{0}^{1} \lambda^{k+1} \sigma(\mathrm{d} \lambda) .
$$

Hence,

$$
\mu(\mathrm{d} \lambda)=\frac{f(\psi) \lambda}{1-\lambda+f(\psi) \lambda} \sigma(\mathrm{d} \lambda) .
$$

In order to satisfy the initial condition, $\psi$ must solve (2.3). Since $f$ is monotone increasing and concave, elementary calculations show that $R(\psi)$ is also monotone increasing and concave. As $R(1)<1$, it follows from the intermediate value theorem that if $f(0)>0$ then (2.3) has a solution. As $R(\psi)$ is concave, this solution is unique. If $f(0)=0$ then $\psi=0$ is one solution to (2.3). By similar arguments, another solution will exist if $R^{\prime}(0)>1$. This last condition can be expressed as inequality (2.4).

\section{A.3. Proof of Theorem 2.3}

Case $(i): \psi>0$. Let $\varepsilon(t, k)=d(t, k)-d(k)$. From (2.1) we have the recursion

$$
\varepsilon(t+1, k)=(1-f(d(t, 0))) \varepsilon(t, k+1)+(f(d(t, 0))-f(\psi))\left(\bar{s}_{k+1}-d(k+1)\right) .
$$

Define $\phi_{t}(z)=\sum_{k=0}^{\infty}|\varepsilon(t, k)| z^{k}$ for all $|z|<1$, and let $c_{\delta}=\sup _{|x-\psi|<\delta} f^{\prime}(x)$ for some $\delta>0$ to be determined. As $\bar{s}_{k} \geq d(k)$ for all $k=0,1, \ldots$, we may bound $\phi_{t}(z)$ by

$$
\begin{aligned}
\phi_{t+1}(z) \leq & (1-f(d(t, 0))) \sum_{k=0}^{\infty}|\varepsilon(t, k+1)| z^{k} \\
& +|(f(d(t, 0))-f(\psi))| \sum_{k=0}^{\infty}\left(\bar{s}_{k+1}-d(k+1)\right) z^{k} \\
\leq & \left(1-f(\psi)+c_{\delta}|\varepsilon(t, 0)|\right) \sum_{k=0}^{\infty}|\varepsilon(t, k+1)| z^{k}+c_{\delta}|\varepsilon(t, 0)| \sum_{k=0}^{\infty}\left(\bar{s}_{k+1}-d(k+1)\right) z^{k} \\
\leq & \frac{1-f(\psi)+c_{\delta}|\varepsilon(t, 0)|}{z}\left(\phi_{t}(z)-|\varepsilon(t, 0)|\right)+c_{\delta}|\varepsilon(t, 0)| \sum_{k=0}^{\infty}\left(\bar{s}_{k+1}-d(k+1)\right) z^{k} \\
\leq & \frac{1-f(\psi)}{z} \phi_{t}(z)+\frac{c_{\delta}}{z} \phi_{t}^{2}(z) \\
& +c_{\delta}|\varepsilon(t, 0)|\left(\sum_{k=0}^{\infty}\left(\bar{s}_{k+1}-d(k+1)\right) z^{k}-\frac{1-f(\psi)}{c_{\delta} z}\right) .
\end{aligned}
$$

We aim to find a $z>(1-f(\psi))$ such that the last term in inequality (A.7) is negative:

$$
\begin{aligned}
\sum_{k=0}^{\infty}\left(\bar{s}_{k+1}-d(k+1)\right) z^{k} & =\int_{0}^{1} \frac{\lambda}{1-\lambda z} \sigma(\mathrm{d} \lambda)-\int_{0}^{1} \frac{\lambda}{1-\lambda z} \frac{f(\psi) \lambda}{1-\lambda+f(\psi) \lambda} \sigma(\mathrm{d} \lambda) \\
& =\int_{0}^{1} \frac{\lambda}{1-\lambda z} \frac{1-\lambda}{1-\lambda+f(\psi) \lambda} \sigma(\mathrm{d} \lambda)
\end{aligned}
$$


Let $z=(1-f(\psi))$. Then

$$
\sum_{k=0}^{\infty}\left(\bar{s}_{k+1}-d(k+1)\right) z^{k}=\frac{R^{\prime}(\psi)}{f^{\prime}(\psi)}<\frac{1}{f^{\prime}(\psi)} .
$$

The above inequality follows since $R^{\prime}(\psi)<1$ if $\psi>0$. Therefore, when $z=(1-f(\psi))$,

$$
\sum_{k=0}^{\infty}\left(\bar{s}_{k+1}-d(k+1)\right) z^{k}-\frac{1-f(\psi)}{f^{\prime}(\psi) z}<0 .
$$

Therefore, from continuity, there exist a $z^{*}>(1-f(\psi))$ and $\delta>0$ sufficiently small such that the last term in inequality (A.7) is negative for $z=z^{*}$. Hence, from inequality (A.7) we obtain

$$
\phi_{t+1}\left(z^{*}\right) \leq \frac{1-f(\psi)}{z^{*}} \phi_{t}\left(z^{*}\right)+\frac{c_{\delta}}{z^{*}} \phi_{t}^{2}\left(z^{*}\right) .
$$

We can conclude that, for sufficiently small $\phi_{0}\left(z^{*}\right), \phi_{t}\left(z^{*}\right) \rightarrow 0$ and, hence, $\psi>0$ is a stable fixed point.

Case (ii): $\psi=0$ and inequality (2.4) holds. In this case $f(0)=0, f^{\prime}(0)>0$, and $d(k)=0$ for all $k=0,1,2, \ldots$ Let $\phi_{t}(1)=\sum_{k=0}^{\infty} d(t, k)$. From (2.1) and as $f$ is concave,

$$
\begin{aligned}
\phi_{t+1}(1) & =\phi_{t}(1)-d(t, 0)+f(d(t, 0))\left(\sum_{k=0}^{\infty} \bar{s}_{k+1}-\phi_{t}(1)+d(t, 0)\right) \\
& \leq \phi_{t}(1)+d(t, 0)\left(f^{\prime}(0) \int_{0}^{1} \frac{\lambda}{1-\lambda} \sigma(\mathrm{d} \lambda)-1\right)+f(d(t, 0))\left(d(t, 0)-\phi_{t}(1)\right) .
\end{aligned}
$$

As inequality (2.4) holds,

$$
\phi_{t+1}(1) \leq \phi_{t}(1)+f(d(t, 0))\left(d(t, 0)-\phi_{t}(1)\right) .
$$

As $d(t, 0)<\phi_{t}(1)$, it follows from inequality (A.8) that $\phi_{t}(1)$ is decreasing in $t$ and, hence, $\lim _{t \rightarrow \infty} \phi_{t}(1)$ exists. Suppose that $\limsup _{t \rightarrow \infty} d(t, 0)=d>0$. Then $\lim _{t \rightarrow \infty} \phi_{t}(1)=$ $\phi_{\infty} \geq d$. For any $\varepsilon>0$, there exists a $T$ such that, for infinitely many $t \geq T, d-d(t, 0)<\varepsilon$ and, for all $t \geq T, \phi_{t}(1)-\phi_{\infty}<\varepsilon$. Therefore, for some $t \geq T$,

$$
\phi_{t+1}(1)-\phi_{t}(1) \leq f(d(t, 0))\left(d-\phi_{\infty}\right)+\varepsilon
$$

and so there exists a finite, positive constant $C$ such that $\phi_{\infty}-d \leq C \varepsilon$. As $\varepsilon$ is arbitrary, this inequality can only hold if $d=\phi_{\infty}$. Hence, $\lim _{t \rightarrow \infty} d(t, k)=0$ for all $k \geq 1$. Finally, we need to show that $\lim _{t \rightarrow \infty} d(t, 0)=0$. We can rewrite recursion (2.1) as

$$
d(t+1,0)=\delta_{t}+f(d(t, 0)) \bar{s}_{1} \leq \delta_{t}+f^{\prime}(0) \bar{s}_{1} d(t, 0),
$$

where $\delta_{t}$ is a positive, bounded sequence converging to 0 . If we can show that $f^{\prime} \bar{s}_{1}<1$ then it will follow that $\lim _{t \rightarrow \infty} d(t, 0)=0$, as required. Since inequality (2.4) holds,

$$
f^{\prime}(0) \bar{s}_{1} \leq f^{\prime}(0) \int_{0}^{1} \frac{\lambda}{1-\lambda} \sigma(\mathrm{d} \lambda) \leq 1 .
$$

If $f^{\prime}(0) \bar{s}_{1}=1$ then

$$
f^{\prime}(0) \int_{0}^{1} \lambda\left(\frac{1}{1-\lambda}-1\right) \sigma(\mathrm{d} \lambda)=0 .
$$

Equation (A.9) implies that $\sigma$ must be supported on $\{0\}$, which is a contradiction since $\bar{s}_{1}>0$. We have now shown that $f^{\prime}(0) \bar{s}_{1}<1$ and this completes the proof. 


\section{A.4. Proof of Theorem 3.1}

Let $\tilde{S}_{t}^{n}=\left\{s_{i}: \tilde{X}_{i, t}^{n}=1\right\}$, where $\tilde{X}_{t}^{n}$ denotes the state of the metapopulation after the colonisation phase at time $t$. We calculate the probability generating functional for $S_{t+1}^{n}$ :

$$
\begin{aligned}
G_{S_{t+1}^{n}}(\xi) & =\mathrm{E}\left(\mathrm{E}\left(\prod_{s_{i} \in S_{t+1}^{n}} \xi\left(s_{i}\right) \mid \tilde{S}_{t}^{n}\right)\right) \\
& =\mathrm{E}\left(\mathrm{E}\left(\prod_{s_{i} \in \tilde{S}_{t}^{n}}\left(Z_{i} \xi\left(s_{i}\right)+1-Z_{i}\right) \mid \tilde{S}_{t}^{n}\right)\right) \\
& =\mathrm{E}\left(\prod_{s_{i} \in \tilde{S}_{t}^{n}}\left(1+s_{i}\left(\xi\left(s_{i}\right)-1\right)\right)\right),
\end{aligned}
$$

where the $Z_{i}$ are independent Bernoulli random variables with $\mathrm{P}\left(Z_{i}=1\right)=s_{i}$. The second equality follows as the probability of patch $i$ surviving the extinction phase is $s_{i}$ and the extinction events at each patch are independent. Now let $\eta(s)=1-s+s \xi(s)$. Then

$$
\begin{aligned}
G_{S_{t+1}^{n}}(\xi) & =\mathrm{E}\left(\mathrm{E}\left(\prod_{s_{i} \in \tilde{S}_{t}^{n}} \eta\left(s_{i}\right) \mid S_{t}^{n}\right)\right) \\
& =\mathrm{E}\left(\left\{\prod_{s_{i} \in S_{t}^{n}} \eta\left(s_{i}\right)\right\} \mathrm{E}\left(\prod_{s_{i} \in \tilde{S}_{t}^{n} \backslash S_{t}^{n}} \eta\left(s_{i}\right) \mid S_{t}^{n}\right)\right) \\
& =\mathrm{E}\left(\left\{\prod_{s_{i} \in S_{t}^{n}} \eta\left(s_{i}\right)\right\} \mathrm{E}\left(\prod_{i \notin S_{t}^{n}}\left(W_{i} \eta\left(s_{i}\right)+1-W_{i}\right) \mid S_{t}^{n}\right)\right),
\end{aligned}
$$

where the $W_{i}$ are conditionally independent Bernoulli random variables with $\mathrm{P}\left(W_{i}=1\right)=$ $f\left(m_{t}^{n} / n\right)$ and $m_{t}^{n}$ is the number of points in $S_{t}^{n}$. The probability generating functional of $S_{t+1}^{n}$ can then be expressed as

$$
\begin{aligned}
G_{S_{t+1}^{n}}(\xi) & =\mathrm{E}\left(\left\{\prod_{s_{i} \in S_{t}^{n}} \eta\left(s_{i}\right)\right\} \exp \left(\sum_{i \notin S_{t}^{n}} \log \left(1+f\left(\frac{m_{t}^{n}}{n}\right)\left(\eta\left(s_{i}\right)-1\right)\right)\right)\right) \\
& =\mathrm{E}\left(\left\{\prod_{s_{i} \in S_{t}^{n}} \eta\left(s_{i}\right)\right\} \exp \left(f^{\prime}(0) \frac{m_{t}^{n}}{n} \sum_{i \notin S_{t}^{n}}\left(\eta\left(s_{i}\right)-1\right)+o_{n}(1)\right)\right) \\
& =\mathrm{E}\left(\left\{\prod_{s_{i} \in S_{t}^{n}} \eta\left(s_{i}\right)\right\} \exp \left(f^{\prime}(0) \frac{m_{t}^{n}}{n} \sum_{i}\left(\eta\left(s_{i}\right)-1\right)+O\left(\frac{m_{t}^{n}}{n}\right)+o_{n}(1)\right)\right) \\
& =\mathrm{E}\left(\prod_{s_{i} \in S_{t}^{n}} \eta\left(s_{i}\right) \exp \left(f^{\prime}(0) n^{-1} \sum_{i}\left(\eta\left(s_{i}\right)-1\right)+O\left(n^{-1}\right)\right)\right) .
\end{aligned}
$$

The final part of the proof proceeds by induction on $t$. We have assumed that $S_{0}^{n}$ is fixed for all $n$ and, hence, it converges weakly to $S_{0}$. Assume that $S_{T}^{n}$ converges weakly to $S_{T}$. The 
probability generating functional of $S_{T+1}$ is given by

$$
\begin{aligned}
G_{S_{T+1}}(\xi) & =\lim _{n \rightarrow \infty} G_{S_{T+1}^{n}}(\xi) \\
& =\lim _{n \rightarrow \infty} \mathrm{E}\left(\prod_{S_{i} \in S_{T}^{n}} \eta\left(s_{i}\right) \exp \left(f^{\prime}(0) n^{-1} \sum_{i}\left(\eta\left(s_{i}\right)-1\right)+O\left(n^{-1}\right)\right)\right) \\
& =\lim _{n \rightarrow \infty} G_{S_{T}^{n}}\left(h_{n}\right),
\end{aligned}
$$

where

$$
h_{n}(s)=(1-s+s \xi(s)) \exp \left(f^{\prime}(0) n^{-1} \sum_{i} s_{i}\left(\xi\left(s_{i}\right)-1\right)+O\left(n^{-1}\right)\right) .
$$

For any suitable $\xi, h_{n}$ converges pointwise to

$$
(1-s+s \xi(s)) \exp \left(f^{\prime}(0) \int_{0}^{1}(\xi(\lambda)-1) \lambda \sigma(\mathrm{d} \lambda)\right) .
$$

Applying Proposition 11.1.VIII and a variant of Theorem 9.4.V from [9], we can conclude that

$$
G_{S_{T+1}}(\xi)=\lim _{n \rightarrow \infty} G_{S_{T}^{n}}\left(h_{n}\right)=G_{S_{T}}\left((1-x+x \xi) \exp \left(-f^{\prime}(0) \int_{0}^{1}(1-\xi(\lambda)) \lambda \sigma(\mathrm{d} \lambda)\right)\right),
$$

and, hence, $S_{t}^{n}$ converges weakly to $S_{t}$ with probability generating function given by (3.1).

\section{A.5. Proof of Theorem 3.2}

Define the functions $q_{t, b}(x)$ by the recurrence relation

$$
q_{t+1, b}(x)=\left(1-x+x q_{t, b}(x)\right) \exp \left(-f^{\prime}(0) \int_{0}^{1}\left(1-q_{t, b}(\lambda)\right) \lambda \sigma(\mathrm{d} \lambda)\right),
$$

subject to the initial condition $q_{0, b}(x)=b$. From (A.10) and Theorem 3.1, the probability of the point process at time $t$ being the empty set is given by $\lim _{b \rightarrow 0} G_{S_{0}}\left(q_{t, b}\right)$. We can show by induction from (A.10) that $q_{t, b}(x) \in[0,1]$ for all $x \in[0,1]$ provided $b \in[0,1]$ and that $\lim _{b \rightarrow 0} q_{t, b}(x)=q_{t}(x)$ pointwise for all $x \in[0,1]$. Applying the dominated convergence theorem, it follows that $\lim _{b \rightarrow 0} G_{S_{0}}\left(q_{t, b}\right)=G_{S_{0}}\left(q_{t}\right)$, where $q_{t}$ denotes $q_{t, 0}$. Since the functions $q_{t}$ are bounded by 1 , again applying the dominated convergence theorem gives

$$
\lim _{t \rightarrow \infty} \mathrm{P}\left(S_{t}=\varnothing\right)=\lim _{t \rightarrow \infty} G_{S_{0}}\left(q_{t}\right)=G_{S_{0}}\left(q_{\infty}\right),
$$

provided $q_{t}(x)$ converges pointwise to $q_{\infty}(x)$ for all $x \in[0,1]$. If $q_{\infty}(x)=1$ then $S_{t}$ will converge to the empty set with probability 1 since $G(1)=1$ for any point process. It remains to investigate the pointwise convergence of $q_{t}(x)$ as $t \rightarrow \infty$.

Define

$$
\psi_{t}=\exp \left(-f^{\prime}(0) \int_{0}^{1}\left(1-q_{t}(\lambda)\right) \lambda \sigma(\mathrm{d} \lambda)\right)
$$

Since

$$
q_{t+1}(x)-q_{t}(x)=\psi_{t-1} x\left(q_{t}(x)-q_{t-1}(x)\right)+\left(\psi_{t}-\psi_{t-1}\right)\left(1-x+x q_{t}(x)\right)
$$


and $q_{1}(x) \geq 0$, it follows that $q_{t}(x)$ is an increasing sequence in $t$ for all $x \in[0,1]$. The sequence $\left\{\psi_{t}\right\}_{t=0}^{\infty}$ is an increasing bounded sequence and, hence, its limit exists. Let $\psi_{\infty}=\lim _{t \rightarrow \infty} \psi_{t}$, and define the function

$$
q_{\infty}(x)=\frac{\psi_{\infty}(1-x)}{1-\psi_{\infty} x} .
$$

Noting that $q_{\infty}(x)=\psi_{\infty}\left(1-x+x q_{\infty}(x)\right)$, we obtain

$$
q_{\infty}(x)-q_{t+1}(x)=\psi_{t} x\left(q_{\infty}(x)-q_{t}(x)\right)+\left(\psi_{\infty}-\psi_{t}\right)\left(1-x+x q_{\infty}(x)\right) .
$$

Since $\psi_{t} \rightarrow \psi_{\infty}$, it follows from (A.12) that $\lim _{t \rightarrow \infty} q_{t}(x)=q_{\infty}(x)$ for all $x \in[0,1]$. By applying the dominate convergence theorem to (A.11), we find that $\psi_{\infty}$ is a solution to the equation

$$
\psi=\exp \left(-f^{\prime}(0) \int_{0}^{1} \frac{(1-\psi) \lambda}{1-\psi \lambda} \sigma(\mathrm{d} \lambda)\right) .
$$

Let $Q(\psi)$ denote the right-hand side of (A.13). The function $Q(\psi)$ is increasing and concave on $[0,1]$. Hence, (A.13) has at most two solutions on $[0,1]$. One solution is obviously $\psi=1$. This is the only solution in $[0,1]$ if $Q^{\prime}(1) \leq 1$. By differentiating $Q$ we see that $Q^{\prime}(1) \leq 1$ is equivalent to (2.4). Now suppose that $Q^{\prime}(1)>1$. Then a second solution to (A.13) exists in $[0,1)$. Denote this solution by $\psi_{*}$, and define

$$
q_{*}(x)=\frac{\psi_{*}(1-x)}{1-\psi_{*} x} .
$$

We can replace $q_{\infty}(x)$ by $q_{*}(x)$ in (A.12) and apply induction to show that $q_{t}(x) \leq q_{*}(x)$. In particular, $\psi_{t} \leq \psi_{*}$ for all $t$. Finally, as $\psi_{\infty}$ must solve (A.13), it follows that $q_{\infty}(x)=q_{*}(x)$.

\section{Acknowledgements}

We are grateful to the referee for helpful comments and suggestions. This research is supported by the Australian Research Council Centre of Excellence for Mathematics and Statistics of Complex Systems.

\section{References}

[1] Abramowitz, M. and Stegun, I. A. (eds) (1965). Handbook of Mathematical Functions with Formulas, Graphs, and Mathematical Tables. Dover Publications, New York.

[2] Andersson, H. and Djehiche, B. (1998). A threshold limit theorem for the stochastic logistic epidemic. J. Appl. Prob. 35, 662-670.

[3] Andersson, M. (1999). The asymptotic final size distribution of multitype chain-binomial epidemic processes. Adv. Appl. Prob. 31, 220-234.

[4] Arrigoni, F. (2003). Deterministic approximation of a stochastic metapopulation model. Adv. Appl. Prob. 35, 691-720.

[5] Buckley, F. M. And Pollett, P. K. (2010). Analytical methods for a stochastic mainland-island metapopulation model. Ecol. Modelling 221, 2526-2530.

[6] Buckley, F. M. ANd Pollett, P. K. (2010). Limit theorems for discrete-time metapopulation models. Prob. Surveys 7, 53-83

[7] Chan, C. K. (2009). Limiting conditional distribution of continuous time Markov chains and their application to metapopulation model. Masters Thesis, University of Queensland.

[8] Cornell, S. J. and Ovaskainen, O. (2008). Exact asymptotic analysis for metapopulation dynamics on correlated dynamic landscapes. Theoret. Pop. Biol. 74, 209-225.

[9] Daley, D. J. And Vere-Jones, D. (2008). An Introduction to the Theory of Point Processes, Vol. II, 2nd edn. Springer, New York. 
[10] Daley, D. J., Gani, J. And YaKowitz, S. (2000). An epidemic with individual infectivities and susceptibilities. Math. Comput. Modelling 32, 155-167.

[11] Darling, R. W. R. AND Norris, J. R. (2008). Differential equation approximations for Markov chains. Prob. Surveys 5, 37-79.

[12] Gani, J. and Stals, L. (2004). The spread of a viral infection in a plantation. Environmetrics 15, 555-560.

[13] Hanski, I. And Gilpin, M. E. (1997). Metapopulation Biology: Ecology, Genetics and Evolution. Academic Press, San Diego.

[14] Hanski, I. and Ovaskainen, O. (2003). Metapopulation theory for fragmented landscapes. Theoret. Pop. Biol. 64, 119-127.

[15] Hill, M. F. and Caswell, H. (2001). The effects of habitat destruction in finite landscapes: a chain-binomial metapopulation model. Oikos 93, 321-331.

[16] Mollanen, A. (2004). SPOMSIM: software for stochastic patch occupancy models of metapopulation dynamics. Ecol. Modelling 179, 533-550.

[17] Ovaskainen, O. (2001). The quasistationary distribution of the stochastic logistic model. J. Appl. Prob. 38, 898-907.

[18] Ovaskainen, O. and Cornell, S. J. (2006). Asymptotically exact analysis of stochastic metapopulation dynamics with explicit spatial structure. Theoret. Pop. Biol. 69, 13-33.

[19] Pellet, J. et al. (2007). An empirical evaluation of the area and isolation paradigm of metapopulation dynamics. Biol. Conservation 136, 483-495.

[20] Preston, C. J. (1977). Spatial birth-and-death processes. Bull. Internat. Statist. Inst. 46, 371-391.

[21] Ter Braak, C. J. F. and Etienne, R. S. (2003). Improved Bayesian analysis of metapopulation data with an application to a tree frog metapopulation. Ecology 84, 231-241.

[22] Weiss, G. H. AND Dishon, M. (1971). On the asymptotic behavior of the stochastic and deterministic models of an epidemic. Math. Biosci. 11, 261-265. 